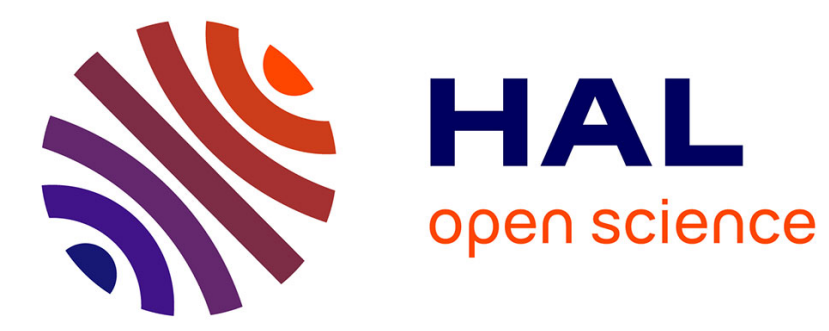

\title{
Is Recycling a Threat or an Opportunity for the Extractor of an Exhaustible Resource?
}

Bocar Samba Ba, Philippe Mahenc

\section{To cite this version:}

Bocar Samba Ba, Philippe Mahenc. Is Recycling a Threat or an Opportunity for the Extractor of an Exhaustible Resource?. Environmental and Resource Economics, 2019, 73 (4), pp.1109-1134. 10.1007/s10640-018-0293-1 . hal-01905045

\section{HAL Id: hal-01905045 \\ https://hal.science/hal-01905045}

Submitted on 28 Oct 2021

HAL is a multi-disciplinary open access archive for the deposit and dissemination of scientific research documents, whether they are published or not. The documents may come from teaching and research institutions in France or abroad, or from public or private research centers.
L'archive ouverte pluridisciplinaire HAL, est destinée au dépôt et à la diffusion de documents scientifiques de niveau recherche, publiés ou non, émanant des établissements d'enseignement et de recherche français ou étrangers, des laboratoires publics ou privés. 


\title{
Is Recycling a Threat or an Opportunity for the Extractor of an Exhaustible Resource?*
}

\author{
Bocar Samba $\mathrm{Ba}^{\dagger} \quad$ Philippe Mahenc ${ }^{\ddagger}$
}

October 4, 2018

\begin{abstract}
We examine the best extraction strategies for the provider of an exhaustible resource that can be recycled. In a two-period model of resource extraction, the extractor faces prospective entry by a recycler that incurs a fixed cost to produce a perfect substitute of the virgin resource. Its entry is an opportunity or a threat for the extractor, depending on whether it maximizes social welfare or its own revenue. Our results highlight how prospective recycling modifies the Hotelling rule.

We characterize various entry possibilities. The benevolent extractor may accommodate or promote recycling, while the self-interested extractor may accommodate or deter recycling.

Keywords: entry, exhaustible resource, Hotelling rule, Ramsey formula, recycling.

JEL Code: D40, L13, Q30.
\end{abstract}

${ }^{*}$ We are grateful to a co-editor and two anonymous referees for their insightful comments and suggestions. This research received funding from the French Agence Nationale de la Recherche within the framework of the project "GREENGO - New Tools for Environmental Governance: the role of NGOs" (ANR-15-CE05-0008). Financial support from the program "Investissements d'Avenir" of the French government (ANR-10-LABX14-01 ) is gratefully acknowledged, too.

${ }^{\dagger}$ Université Clermont Auvergne, CNRS, CERDI, F-63000 Clermont-Ferrand, France, E-mail: bocar_samba.ba@uca.fr

${ }_{\mp}$ CEE-M, Univ Montpellier, CNRS , INRA, Montpellier SupAgro, Montpellier, France, Avenue Raymond Dugrand, Site de Richter, C. S. 79606, 34960 Montpellier Cedex 2 - France - Email: philippe.mahenc@umontpellier.fr 


\section{Introduction}

Recycling is a fast-growing industry in a world where the issue of resource conservation is becoming more acute. For instance, Baksi and Long (2009) point out that the percentage of municipal solid waste recycled in the United States has increased almost fivefold between 1960 and 2007. The question as to whether recycling conserves resources is particularly relevant with regard to exhaustible resources that can be partially recycled after use.

In an early analysis of the issue, Weinstein and Zeckhauser (1974) show that perfectly competitive markets achieve a socially efficient consumption stream that involves, after an initial period of extraction only, a number of periods in which the virgin and the recycled resources coexist. The authors conclude their analysis by wondering whether imperfect competition might lead to too rapid a rate of resource consumption with recycling. That question, though important, has been left unanswered. The providers of exhaustible resources, such as phosphorus or aluminum, are endowed with significant market power (see the references in the next section). For these resources, technological progress over the last decades has yielded less costly recycling procedures that provide consumers with close substitutes for the virgin resource. For instance, in Germany the costs of recycling phosphorus have declined over the past decade and, given the price for phosphate fertilizers from rock phosphate, some recycling technologies can now be profitably operated (Sartorius 2011).

In this paper, we examine the strategic interplay between the extractor of an exhaustible resource and the recycler of this resource. How do the extractors influence the amount of recycled material that they will later have to compete with? Does the prospect of recycling accelerate or slow down the depletion of exhaustible resources? Our purpose is to provide a simple theoretical insight into these issues.

As a benchmark, we analyze a two-period model of resource extraction, in which the extractor of the virgin resource and the recycler are integrated into a single entity that maximizes welfare, like a social planner, under the break-even constraint of non-negative profits from recycling. Our preliminary findings extend Weinstein and Zeckhauser's (1974) predictions that recycling per se may have an impact on the optimal extraction of resources, independently of strategic considerations. We show that the possibility of recycling increases the initial extraction of the virgin resource more than predicted by the Hotelling rule, to make sure that the social cost of recycling is covered.

The core issue of the paper is to investigate a two-period model with the assumption that the extractor and the recycler are separate entities. Faced with the prospective entry of the recycler in the second period, the extractor chooses how much of the virgin resource is extracted in the first period. We assume that the recycler maximizes its profit and incurs a fixed cost to enter the marketplace. This entry is an opportunity or a threat for the extractor, depending on whether it maximizes social welfare or its own revenue. Therefore, our analysis distinguishes between the two cases.

The extractor anticipates how its initial choice affects the present and future demands 
for the resource, hence the market profitability in the second period. By virtue of providing the input for the recycler, the extractor can exploit its first-mover advantage to signal its post-entry behavior to the recycler, thereby influencing its entry decision. To be successful, this signal must be credible, which may entail a cost for the extractor. This opens up a rich set of entry possibilities and our findings highlight how prospective recycling modifies the Hotelling rule for each equilibrium outcome.

When the extractor maximizes social welfare, it views recycling as a desirable activity because recycling creates a valuable expansion of the resource stock. This scale effect has been previously recognized by André and Cerdá (2006). As a result, the benevolent extractor accelerates the resource depletion to enhance the market position of the recycler, thereby improving the profitability of recycling. Depending on the size of the fixed cost of recycling, there are two entry possibilities. If the fixed cost is low, then the extractor accommodates the recycler's entry by providing more input than needed to cover the costs of recycling. If the fixed cost is high, then the extractor promotes recycling by providing just enough input to offset the costs of recycling. In that case, the extractor must incur a cost to signal that recycling is worthwhile by increasing initial extraction above the accommodating level.

When the extractor maximizes its own profit, it views recycling as a threat to its market position. In that case, we find that the extractor must slow down the resource depletion for two different reasons depending on the size of the fixed cost of recycling. If the fixed cost is low, then the extractor can do no better than accommodate the recycler's entry. In this equilibrium, the extractor accepts some output reduction in the first period to defend its market position in the second period by triggering a less aggressive reaction from the recycler. For higher values of the fixed cost, the extractor deters recycling by signaling its intention to flood the market with virgin product in the second period, so that the market becomes unattractive to the recycler. Due to limit pricing, this signal proves costly for the extractor, which finally makes it credible too.

The rest of this paper is organized as follows. Section 2 presents a detailed review of the related literature. Section 3 introduces the two-period model. Section 4 presents the optimal outcome in the case of a social planner making both the extraction and the recycling decisions. In Section 5, we analyze the case in which the extractor and the recycler are two independent players. Our concluding remarks appear in Section 6.

\section{Motivation and related literature}

The history of exhaustible resources provides evidence that the extraction sector goes through various regimes of competition and the recycling market is often ill-organized. Martin (1982) recognizes that "many of the industries currently practicing recycling are highly concentrated".

One interesting example is phosphate extraction together with phosphorus recycling. The majority of global phosphate rock reserves are located in Morocco, providing this country 
with a monopoly position in supplying the virgin resource (see Cordell et al. 2009). Thus, one may expect governmental regulation in Morocco to play a leading role in choosing the amount of virgin phosphate to be extracted. In turn, this regulation may be more or less benevolent, depending on various factors, such as the pressure put on the government by the shareholders of the extraction company, or the share of the consumer surplus that escapes the government's jurisdiction. At the same time, the sector of phosphorus recycling has no institutional or organizational home (Cordell et al. 2009; Livingston et al. 2005). Throughout the world, phosphorus recycling is mainly based on the reuse of nutrient flows stemming from food production and consumption. ${ }^{1}$ While the sanitation sector in cities, such as waste water treatment or sewage sludge plants, plays a key role in phosphorus recycling ${ }^{2}$, this service is scarcely high on the agenda of extraction stakeholders. In addition, the process of recycling phosphorus from sewage or waste water often requires a specific infrastructure and high levels of technical skills. According to Weikard and Seyhan (2009), phosphorus recycling is mainly undertaken by developed countries, except for Pakistan, not only because they have advanced wastewater treatment technologies but also because, unlike developing countries, they have phosphorus-saturated soils.

Weikard and Seyhan (2009), and Seyhan et al. (2012) investigate the effect of recycling on the depletion of phosphorus in a competitive model where developed countries control both resource extraction and recycling. They show that the Hotelling rule applies and recycling delays the depletion of phosphorus. This framework is similar to the benchmark case that we investigate in Section 4 of the present paper, where the extractor of the virgin resource and the recycler are integrated into a single entity that maximizes welfare like a social planner. We assume further that recycling involves large fixed costs, which might cause deficits under marginal cost pricing. We conclude that the Hotelling rule applies only when the social cost of financing the recycling activity is nil. When it is positive, the rate of increase of the resource price must instead exceed the rate of interest.

Another example of a recyclable exhaustible resource is aluminum. This is now well documented because aluminum has been recycled since the early $1900 \mathrm{~s}^{3}$ The monopolistic nature of virgin aluminum production in 1945 was acknowledged by the famous Alcoa case (Swan 1980). ${ }^{4}$ In contrast, the recycling sector of the industry is generally considered to

\footnotetext{
${ }^{1}$ There are various methods available to recover phosphorus, such as ploughing crop residues back into the soil, composting food waste from households, using human and animal excreta, and so on.

2 "Around $41 \%$ of phosphorus from sewage sludge across the European Union is currently recovered and reused in agriculture", from the European Commission's expert seminar on the sustainability of phosphorus resources (2011, http://ec.europa.eu/environment/natres/pdf/conclusions_17_02_2011.pdf.). Even now, according to Ensink et al. (2004), more than $25 \%$ of urban vegetables grown in Pakistan are being fertilized with municipal wastewater.

${ }^{3}$ In 1989 , about $28 \%$ of the total aluminum supply in the United States came from recycled aluminum (see http://www.epa.gov/osw/nonhaz/municipal/pubs/sw90077a.pdf).

${ }^{4}$ In 1945, Alcoa was judged to enjoy a strong monopoly position, which was supported rather than threatened by competition from secondary aluminum produced by recycling scrap aluminum. Swan (1980) provides empirical evidence that the price charged by Alcoa is only slightly below the pure monopoly price but is well above the purely competitive price. The question of whether Alcoa had maintained its monopoly position by strategically controlling the supply of scrap aluminum that was ultimately available to secondary producers
} 
be competitive throughout the literature. In the view of Friedman (1967), the competitive recycler would tend to push the aluminum price down to the marginal cost of virgin aluminum production. Martin (1982), Suslow (1986), and Grant (1999) dispute this statement under the assumption that Alcoa was confronted by an independent and competitive recycling sector. Their results generally show that recycling barely eroded Alcoa's market power. Since then, the aluminum industry has gone through different regimes of imperfect competition, both in the extraction and the recycling sectors.

The early theoretical literature on the extraction of exhaustible resources has examined how market power in the extraction sector affects the Hotelling rule. The present paper extends the scope of this research by allowing for the possibility that recycling yields a perfect substitute for the virgin product. On the one hand, recycling is tantamount to increasing the resource stock, which spurs the extractor to accelerate the resource depletion. On the other hand, recycling by an independent company represents the threat of potential competition from the extractor's standpoint. Our findings mainly contribute to the traditional literature on the Hotelling rule by providing insight into the strategic interplay between the extractor and the recycler. Hotelling (1931) shows that the monopolist has a tendency to be more resource-conservative than "competition... or maximizing of social value would require". Stiglitz (1976) adds that the parsimony of the monopolist depends on the elasticity of demand and extraction costs. Except for the case where the elasticity of demand is constant and extraction costs are zero, the result that the monopolist extracts the resource at a lower rate than that of the competitive firm seems rather robust (see also Tullock 1979) for the case of inelastic demand). Gilbert and Goldman (1978) find that the monopolist may deter the entry of potential competitors by reducing the initial extraction below the level that is optimal when the monopolist is not constrained by entry. In the present setting, the monopolist also discourages the entry of the recycler with a decrease in the initial extraction.

As previously mentioned, the issue of recycling an exhaustible resource was addressed by Weinstein and Zeckhauser (1974), and was more recently developed with the aforementioned debate on the Alcoa case. The benchmark case in Section 4 of the present paper is closely related to Weinstein and Zeckhauser (1974). The difference is that, in our analysis, the social planner uses the proceeds from the sales of the recycled resource to cover the social cost of recycling. Another difference is that demand is divided between resource extraction and recycling in the present paper, while the total supply of the virgin resource is used up before any recycling begins in their analysis. When demand is met entirely by recycling after the resource has been used up, they show that the market price may exceed the marginal cost of recycling due to an excess of demand over supply. In contrast, we show that the final market price for the resource must lie between marginal cost and the monopoly price, depending on the social cost of financing the recycling activity.

Hollander and Lasserre (1988) investigate the case of a monopolist in the extraction sector which recycles the scrap from its own production. The monopolist has monopsony power in has been debated at length in the economic literature. Grant (1999) provides a useful survey of this debate. 
the scrap market and faces a fringe of price-taking recyclers. Consequently, the extractor finds it profitable to pre-empt market entry by competitive recyclers when the cost of recycling is sufficiently high. Entry pre-emption has been shown to increase the resource price above the level that would prevail in the absence of a threat of entry because the extractor must pay a high price for withdrawing the scrap that is available to recyclers from the market. Section 5 of the present paper also considers the case of strategic rivalry between the extractor and the recycler. Our setting differs from that of Hollander and Lasserre (1988) in two respects. First, the extractor does not recycle its own output: competition occurs in the second period where it involves the virgin resource supplied by the extractor and a substitute product supplied by the recycler. Second, the extractor is committed to its initial choice of extraction before recycling occurs. Hence, the first-period extraction signals to the recycler whether or not entry is profitable in the second period, depending on the post-entry behavior of the extractor. To make the recycler's entry unprofitable, the extractor signals its intention to flood the market with the virgin resource in the second period. This raises the first period price above the level that would prevail in the absence of a threat of entry, leading to a lower price in the second period, which makes the market unattractive to the recycler.

Gaudet and Van Long (2003) examine how market power in the recycling industry affects the primary production of an inexhaustible resource. They show that the possibility of recycling may increase the market power of the extractor. Clearly, this cannot occur in the present model because competition between the exhaustible resource and its recycled output mitigates the extractor's market power.

André and Cerdà (2006) analyze recycling as a production technology that transforms waste into recycled materials. They highlight two different impacts of recycling on the growth of the economy: a technological effect, which affects the mixing of renewable and non-renewable inputs in the production process; and a scale effect, which increases the availability of recyclable resources. In the short run, recycling may alleviate resource scarcity and allow for higher output levels but it cannot avoid resource exhaustibility in the long run. In the present setting, the scale effect also plays a key role, especially when the weight of profit in the extractor's objective function is relatively low. In that case, the scale effect strengthens the incentive to extract more of the resource prior to recycling because an increase in the available stock of the resource is socially valuable.

Lastly, Gaudet (2007) and Slade and Thille (2009) provide comprehensive reviews of the literature on the extraction of exhaustible resources. According to them, there is empirical evidence that, for many resources, prices have fallen or remained unchanged over long periods, which is inconsistent with simple versions of Hotelling's model but is not inconsistent with the monopoly formulation of the rule (Stiglitz 1976; Gaudet 2007). The monopoly variant of the model used in the present paper shows that the threat of business stealing by the recycler further flattens the price path of the resource, which may be able to explain falling prices. 


\section{The baseline two-period model}

We consider a two-period exhaustible resource market, in which an extractor has sole extraction rights for the resource. Consumer demand is assumed to be separable by time period. Let the market inverse demand curve in each period be $p_{t}=P_{t}\left(q_{t}\right)$, where $t$ is an index over time periods, $q_{t}$ is the total quantity consumed in period $t$, and $p_{t}$ is the price in that period. To limit the analysis to two periods like, for example, Gaudet et al. (1995), we assume that $p_{t}=0$ for all $t>2$. Let $\delta=\frac{1}{1+\rho}$ denote the common discount factor, where $\rho$ is the rate of interest.

The extractor is endowed with a stock, $s$, and determines the amount $q_{1}=q$ that it will extract and sell at price $p_{1}=P_{1}(q)$ in the first period. The extractor can extract the resource and transport it to the market at no cost. Exploration does not occur and $s$ is the single known stock of the resource. We assume for simplicity that the extractor sells all of the remaining resource stock, $s-q$, in the second period, after which the resource becomes worthless. In the second period, the extractor faces the entry of a recycler. The recycled resource is viewed by consumers as a perfect substitute for the extracted resource. The recycler increases the quantity of the available resource by the amount $r$, and, hence, the market clears at the price $p_{2}=P_{2}\left(q_{2}\right)$ in the second period, with $q_{2}=s-q+r$.

We assume that the first-period consumers dispose of the used resource by giving it to the recycler because it is the only producer capable of recycling part of the resource from used quantities. ${ }^{5}$ The amount recycled $r$ cannot exceed $q$ due to the depreciation and shrinkage that are present in every recycling process. ${ }^{6}$ The recycling process requires the installation of capacity (e.g., sewage plants, specialized machinery for recycling, etc.), which involves a fixed set-up cost $F$. We shall suppose that this cost is incurred up front. The recycling cost function is given by $c(r)$. We make the following assumption on $c(r)$ :

$(A 1) c(r)$ is a non-decreasing, twice continuously differentiable and convex function on $[0, q]$ with $c(0)=0$.

The consumers' gross surplus in period $t$ is $S_{t}\left(q_{t}\right)=\int_{0}^{q_{t}} P_{t}(x) d x$, and we assume that $P_{t}($. is twice continuously differentiable with $P_{t}^{\prime}()<.0 .{ }^{7}$

We make a further assumption on $P_{t}($.$) :$

(A2) $P_{t}($.$) is log-concave. { }^{8}$

\footnotetext{
${ }^{5}$ Regarding phosphorus, for instance, the recycler may be viewed as the group of developed countries with phosphorus-saturated soils and advanced wastewater treatment technologies (see Weikard and Seyhan 2009).

${ }^{6}$ See Martin (1982) for aluminum scrap recycling and Weikard and Seyhan (2009) for phosphorus recycling from sewage sludge.

${ }^{7} P_{t}^{\prime}($.$) denotes the derivative of P_{t}(x)$ with respect to $x$. Throughout the text, the superscripts ' and " will be used to represent, respectively, the first and second derivatives of a function of a single variable.

${ }^{8} \mathrm{~A}$ function $P_{t}($.$) is \log$-concave if $P_{t}^{\prime \prime}(.) P_{t}()-.P_{t}^{\prime}(.)^{2}<0$. This assumption is very general: $\log P$ is
} 
As recycling cannot emerge ex nihilo, we will assume that recycling is too costly to be worthwhile at $q=0$, thus, $P_{2}(s+r)<c^{\prime}(r)$ for all $r \geq 0$. Furthermore, the whole stock of the resource must be large enough to allow for recycling. Hence, at $q=s$ and $r=0$, we must have $P_{2}(s-q+r)>c^{\prime}(r)$. We summarize these assumptions as follows:

(A3) $P_{2}(s)<c^{\prime}(0)<P_{2}(0)$.

The extractor.-The extractor obtains the intertemporal revenue $R(q, r)=P_{1}(q) q+$ $\delta P_{2}(s-q+r)(s-q)$ from extraction. It may be purely self-interested, in which case its objective is to maximize $R(q, r)$. Alternatively, the extractor may have some social concern and maximize the present discounted value of the social welfare, that is, $W(q, r)=S_{1}(q)+$ $\delta S_{2}(s-q+r)$. To distinguish between each of these cases, we will write the extractor's objective function $V(q, r)$ as a convex combination of the social welfare and the revenue from extraction:

$$
V(q, r)=(1-\lambda) W(q, r)+\lambda R(q, r)
$$

where $\lambda$ is a dummy variable that takes the value 0 or 1 to indicate that the extractor is socially benevolent or self-interested.

The case where the extractor is highly driven by a concern for revenue is motivated by real-world features of the phosphorus and aluminum industries. The market for phosphorus is mainly characterized by high concentrations of phosphate reserves in a few countries, such as Morocco and China (see, for example, Cordell et al. 2009 or Weikard and Seyhan 2009). In both of these countries, however, a very large share of the extraction companies are state owned: whether or not they abuse market power depends on the state regulation, if any. The case $\lambda=1$ is also closely related to Swan's (1980) study of the market for aluminum, where the monopolist Alcoa is confronted by an independent competitive recycler (see also Martin 1982). According to Wan and Boyce (2014), the market structure in the extraction of platinum, nickel and diamond is quite concentrated, too.

The recycler.-Our analysis will deal separately with two scenarios of organizational integration of extraction and recycling. In the first scenario, the recycler and the extractor of the virgin resource are integrated into a single entity. This scenario is treated in Section 4 under the assumption that the decision maker maximizes welfare. The outcome will serve as a benchmark that points to the effects of recycling on the optimal extraction of the resource, independently of strategic considerations. In the second scenario, the extractor and the recycler are separate entities engaged in strategic interaction. Section 5 explores this scenario under the assumption that the independent recycler maximizes profit.

In each of the two scenarios, recycling involves a fixed cost, which may lead to deficits under marginal cost pricing. This may impose a break-even constraint of non-negative profits

concave if $P$ is concave, linear or $P(q)=A q^{\gamma-1}$ with $0<\gamma<1$ so that $1 /(1-\gamma)$ is the elasticity of demand. Most of the commonly used demand functions are, in fact, log-concave. The limiting case is $P(q)=A e^{-q}$, which is strictly convex and log-linear (hence log-concave). 
from recycling in both scenarios:

$$
\pi(q, r) \geq 0
$$

where $\pi(q, r)=P_{2}(s-q+r) r-c(r)-F$ is the recycling profit function.

We will denote by $\mu$ the Lagrange multiplier of the constraint (2), regardless of the scenario. If $\mu$ happens to be positive in the equilibrium outcome, then the break-even constraint is binding. An increased value of $\mu$ will indicate that higher proceeds from sales of the recycled resource are needed to cover the growing costs of recycling. Generally, the multiplier $\mu$ measures the social cost of financing the recycling activity.

To illustrate the results obtained in the general setting, we will solve explicitly for the equilibria within a specific framework, in which consumer surplus is quadratic and stationary: $S_{t}\left(q_{t}\right)=a q_{t}-q_{t}^{2} / 2, t=1,2$, so that demand is linear: $P_{t}\left(q_{t}\right)=a-q_{t}, t=1,2$; that variable costs are also linear: $c(r)=c r$, with $a>c$; and that $\delta=1$, hence $\rho=0$.

This framework satisfies assumptions $(A 1)$ and $(A 2)$. Moreover, assumption $(A 3)$ amounts to

$$
a-s<c<a .
$$

and the recycler's profit function becomes

$$
\pi(q, r)=(a-s+q-r) r-c r-F .
$$

\section{The extractor and the recycler are integrated into a single entity}

To begin, we examine the situation in which the extractor and the recycler are integrated, and thus the extraction and the recycling choices are made by a single decision maker. We formulate this problem following the standard approach that was identified by Ramsey (1927), developed by Boiteux (1956), and synthesized by Baumol and Bradford (1970). We assume that the decision maker behaves as a social planner whose goal is to maximize social welfare under the break-even constraint of non-negative profits from recycling. In this scenario, the welfare function is computed net of recycling costs, as indicated by the index $n$ :

$$
W^{n}(q, r)=S_{1}(q)+\delta\left[S_{2}(s-q+r)-c(r)-F\right] .
$$

The solution of the social planner's problem is an initial amount of virgin resource and a recycling output $(\widehat{q}, \widehat{r})$ that maximizes (5) subject to the constraint (2) associated with the Lagrange multiplier $\widehat{\mu}$. In the equilibrium outcome, $\widehat{p}_{1}=P_{1}(\widehat{q})$ and $\widehat{p}_{2}=P_{2}(s-\widehat{q}+\widehat{r})$ will denote the market prices in the first and the second period, respectively. Note that in this problem with a single decision maker, the initial extraction $\widehat{q}$ has no commitment value.

Extraction of the virgin resource provides the input for recycling under the constraint of non-negative profits from recycling. The optimal recycling decision for the social planner is a function $\widehat{r}(q, \mu)$ of the output $q$ and the social cost $\mu$. Assuming an interior solution, $\widehat{r}(q, \mu)$ 
satisfies the necessary condition $W_{r}^{n}(q, r)+\mu \pi_{r}(q, r)=0 .^{9}$

The following lemma states the existence and properties of $\widehat{r}(q, \mu)$.

Lemma 1: Given a market for an exhaustible natural resource with inverse demand $P_{t}($. and recycling costs $c(r)$ satisfying assumptions $(A 1),(A 2)$ and $(A 3)$, there exists a unique local maximum $\widehat{r}(q, \mu)$ such that $0<\widehat{r}_{q}(q, \mu)<1$.

Proof: see Appendix 1.

Given $\mu$, the recycling function $\widehat{r}(q, \mu)$ is upward sloping with respect to $q$ because removing more of the virgin resource from the ground induces the social planner to recycle more in the next period. The inequality $\widehat{r}_{q}(q, \mu)<1$ reflects the fact that recycling is subject to entropy: the process of resource recycling cannot work with $100 \%$ efficiency.

Denoting by $\varepsilon=-\frac{P_{2}(s-q+r)}{P_{2}^{\prime}(s-q+r) r}$ the price elasticity of demand for the recycled resource, we write $\widehat{\varepsilon}$ for the price elasticity given in equilibrium by $(\widehat{q}, \widehat{r})$, where $\widehat{r}=\widehat{r}(\widehat{q}, \widehat{\mu})$.

Proposition 1: When a social planner implements both extraction and recycling under assumptions $(A 1),(A 2)$ and $(A 3)$, the relative profit margin from recycling is inversely proportional to the elasticity of demand for the recycled product and the resource price rises faster than the interest rate. If $\widehat{\varepsilon}>\frac{\widehat{\mu}}{\delta}$, then

$$
\begin{aligned}
\frac{\widehat{p}_{2}-c^{\prime}(\widehat{r})}{\widehat{p}_{2}} & =\frac{\widehat{\mu}}{(\delta+\widehat{\mu})} \frac{1}{\widehat{\varepsilon}}, \\
& \text { and } \\
\frac{\widehat{p}_{2}-\widehat{p}_{1}}{\widehat{p}_{1}}>\rho . &
\end{aligned}
$$

Proof: see Appendix 2.

The equation (6) is the standard Ramsey formula: the pricing of the recycled resource is positively related to the social cost of financing the recycling activity and inversely related to the demand elasticity. When recycling causes no deficit (which corresponds to $\widehat{\mu}=0$ ), the market price for the resource after recycling equals the marginal cost of recycling. This particular result coincides with that obtained by Weinstein and Zeckhauser (1974) in the variant of their model where they assume that a fixed supply of the resource can be recycled at a cost. One difference from Weinstein and Zeckhauser (1974) is that demand is met entirely by recycling after the resource has been used up, whereas in our setting the demand is also met by resource extraction.

In the present setting, we further assume that recycling involves large fixed costs. The social planner must use the proceeds from the sales of the recycled product to cover the social cost of recycling. As a result, the final market price for the resource must lie between its marginal cost and its monopoly price, at a level depending on the elasticity of demand for the recycled resource and the fixed cost of recycling. As $\widehat{\mu}$ grows in equilibrium, the need

\footnotetext{
${ }^{9}$ For a function with several variables, the partial derivative with respect to a variable is denoted by that variable's being written as a subscript.
} 
for budgetary revenue increases and a larger premium above the marginal cost of recycling becomes necessary to cover the cost of recycling. In the limit case where $\widehat{\mu}$ is infinite, the price for the resource after recycling tends to the monopoly price because monetary considerations are weighted infinitely more than the value created by recycling.

The desire to accommodate recycling modifies the Hotelling rule in the following way:

$$
\frac{\widehat{p}_{2}-\widehat{p}_{1}}{\widehat{p}_{1}}=\rho+(1+\rho) \frac{\widehat{\mu}}{\widehat{\varepsilon}} \widehat{p}_{2} .
$$

As usual, the rate of price increase must equal the opportunity cost of deferred extraction. The standard rule of Hotelling applies only when recycling is socially costless $(\widehat{\mu}=0)$, which is consistent with Weinstein and Zeckhauser (1974, pp. 77-78): according to their predictions, the willingness to pay for the use of the virgin resource grows at the rate of interest before recycling begins.

When $\widehat{\mu}>0$, the rate of increase of the price exceeds the rate of interest because the present value of extraction takes further into account the budgetary cost of recycling the extracted resource. This cost includes both the financing of the recycling costs and the cost of raising funds due to pricing above marginal cost. The second term in the right-hand side of (8) represents the foregone revenue in the sale of the recycled output, which depends both on the price elasticity of demand for the recycled resource, and on the overall costs entailed by recycling. In a nutshell, recycling accelerates the depletion of the resource to make sure that the social cost of recycling is covered.

The framework of linear demand and cost functions.-We now derive explicit solutions $(\widehat{q}, \widehat{r})$ in the specific example. With linear demand and cost functions, the welfare function is

$$
W^{n}(q, r)=a q-q^{2} / 2+a(s-q+r)-(s-q+r)^{2} / 2-c r-F,
$$

The recycling profit is given by (4). The recycling function is

$$
\widehat{r}(q, \mu)=\frac{1+\mu}{1+2 \mu}(a-s-c+q) .
$$

Figure 1 shows the isowelfare curves (in black) given by (9) and the isoprofit curves (in gray) given by (4) in $(q, r)$ space. The isowelfare curves are ellipses of the form $\left\{(q, r): W^{n}(q, r)=\mathrm{W}^{n}\right\}$ for some isowelfare level $\mathrm{W}^{n}$. The ellipses are centered at the point $B$ that identifies the maximal welfare point corresponding to the solution of the social planner's problem with no break-even constraint. In that case, $F=0$, thus $\widehat{\mu}=0$ and Proposition 1 yields that $\widehat{p}_{2}=\widehat{p}_{1}=c$ at point $B$, whose coordinates are $(a-c, 2(a-c)-s)$. The existence of a solution at $B$ requires that the resource is moderately abundant, so that $s<2(a-c)$. As the levels of $F$ decrease, the isowelfare levels increase when shifting from $A$ to $B$ along the line defined by $W_{q}^{n}(q, r)=0$, which boils down to $p_{2}=p_{1}$. The line $(C, D)$ cuts each of the isoprofit curves at its maximum, according to the equation $\pi_{r}(q, r)=0$. Thus, profit levels are higher as the isoprofit curves shift to the right along the line $(C, D)$, due to the increasing 


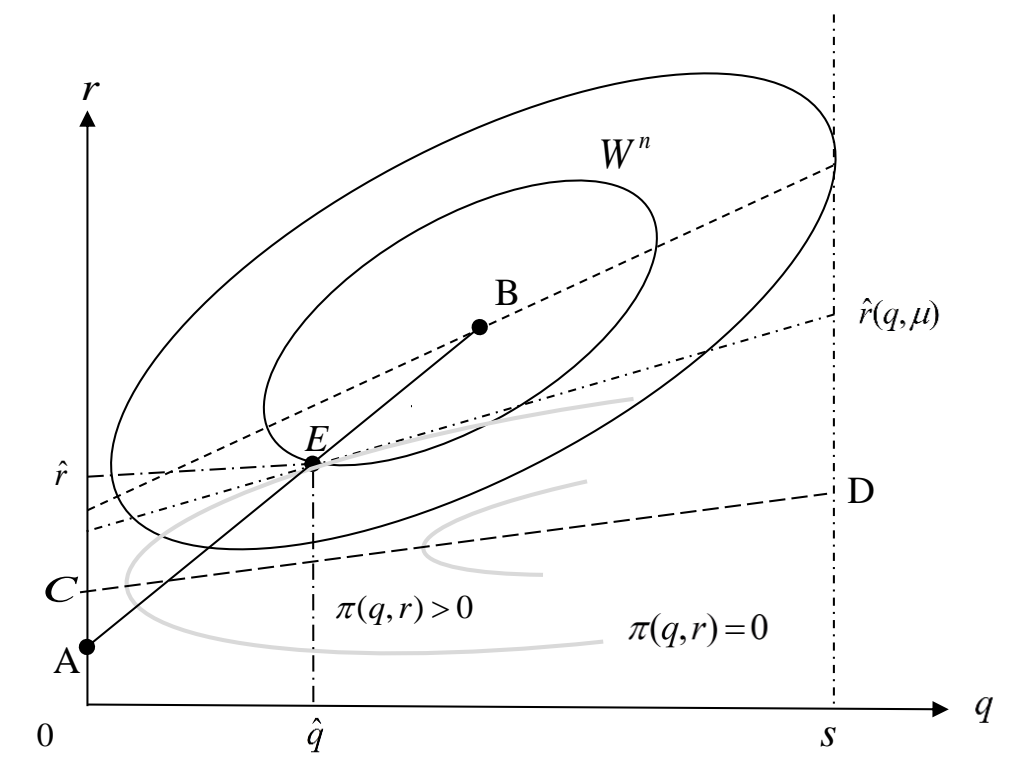

Figure 1: Equilibrium solutions for the social planner 
levels of $F$.

The social planner's problem is to find a location on the segment $[A, B]$ that maximizes welfare given the break-even constraint. The solution occurs at point $E$ in the figure, where $[A, B]$ crosses the set of points such that $\pi(q, r)=0$. Point $E$ has coordinates $(\widehat{q}, \widehat{r})$ characterized by

$$
\begin{aligned}
& \widehat{q}=\frac{1}{4}\left(2(a-c)+s+\sqrt{(2(a-c)-s)^{2}-8 F}\right), \\
& \widehat{r}=\frac{1}{2}\left(2(a-c)-s+\sqrt{(2(a-c)-s)^{2}-8 F}\right),
\end{aligned}
$$

and associated with $\widehat{\mu}$ given by

$$
F=\frac{\widehat{\mu}(1+\widehat{\mu})(2(a-c)-s)^{2}}{\left(1+2 \widehat{\mu}-\widehat{\mu}^{2}\right)^{2}} .
$$

A necessary condition for the existence of $\widehat{q}$ is that $F \leq F^{\circ}=\frac{(2(a-c)-s)^{2}}{8}$. Hence, $F^{\circ}$ is the maximum level of fixed cost below which recycling is socially desirable.

Furthermore, from Equation (12), we have that $\widehat{\mu}$ is increasing with $F$ from $\widehat{\mu}=0$ at $F=0$, to $\widehat{\mu}=1+\sqrt{3}-\sqrt{3+2 \sqrt{3}}$ at $F=F^{\circ}$ under the restriction that $1+2 \widehat{\mu}-\widehat{\mu}^{2}>0 .{ }^{10}$ This shows that increasing fixed costs of recycling raise the social cost of financing the recycling activity $\widehat{\mu}$ because higher proceeds from sales are needed to cover the cost of recycling.

The following corollary summarizes the result obtained with linear demand and cost functions.

Corollary 1: Assume that $s<2(a-c)$ and $F<F^{\circ}$ within the framework of linear demand and cost functions. The social planner achieves an optimal outcome characterized by (11a) and (11b).

\section{The extractor and the recycler are separate entities}

In the outcome of the social planner's problem, the initial extraction of the virgin resource has no commitment value. In contrast, when the extraction and the recycling decisions are made by two independent entities, the extractor is committed to its initial choice of extraction, which provides the input for the recycler. De facto, the extractor cannot change the initial extraction for the period during which the recycler makes its choice. To formalize the idea that the recycler responds to the extractor's initial choice after some lag, we study a twoperiod model with the following timing. In the first period, the extractor chooses $q$. In the second period, the recycler observes $q$, responds to that action by choosing the output $r$, and the extractor simultaneously sells $s-q$.

\footnotetext{
${ }^{10}$ We need this restriction because $\widehat{q}=\frac{(1+\widehat{\mu})^{2}(a-c)-s \widehat{\mu}^{2}}{1+2 \widehat{\mu}-\widehat{\mu}^{2}}$ and $\widehat{r}=\frac{(1+\widehat{\mu})(2(a-c)-s)}{1+2 \widehat{\mu}-\widehat{\mu}^{2}}$.
} 
In this scenario, we assume that the extractor maximizes the objective function (1) under the break-even constraint (2). The notation $\mu$ for the Lagrange multiplier associated with this constraint is still valid. We also assume that the recycler maximizes profit. The extractor chooses a point on the recycler's reaction function to maximize its own objective function. A (pure) strategy for the extractor is a quantity $q$ and a strategy for the recycler is a mapping $r(q):[0, s] \rightarrow[0, s]$. The equilibrium of the two-period game reduces to a pair $\left(q^{*}, r^{*}().\right)$ associated with the multiplier $\mu^{*}$ such that:

(i) $r^{*}(q)=\arg \max _{r \in[0, s]} \pi(q, r)$ subject to $\pi\left(q, r^{*}(q)\right) \geq 0$,

(ii) $q^{*}=\arg \max _{q \in[0, s]} V\left(q, r^{*}(q)\right)$.

In the equilibrium outcome, we will generally write $p_{1}^{*}=P_{1}\left(q^{*}\right)$ and $p_{2}^{*}=P_{2}\left(s-q^{*}+r^{*}\left(q^{*}\right)\right)$ for the first- and the second-period market prices, respectively. Moreover, we will denote the price elasticities of demand for the virgin product in the first and the second period, respectively, by $\eta_{1}=-\frac{p_{1}}{P_{1}^{\prime}(.) q}$ and $\eta_{2}=-\frac{p_{2}}{P_{2}^{\prime}(.)(s-q)}$. Hence, we will write $\eta_{t}^{*}, t=1,2$, for the equilibrium values of the demand elasticity in each period for the virgin product, and $\varepsilon^{*}$ for the equilibrium value of the demand elasticity for the recycled resource.

In this setting, the extractor anticipates how its initial choice of extraction affects not only the present and future demands for the resource but also the profitability of the market for the recycler. Unlike the social planner in the previous section, the extractor now behaves strategically. The initial extraction "signals" the extractor's post-entry behavior to the recycler. Clearly, this signal influences the recycler's view of what will happen if it enters, thereby making entry more or less difficult. The extractor's willingness to accommodate the recycler's entry depends on whether the extractor is socially benevolent or self-interested. If the extractor maximizes social welfare $(\lambda=0)$, it is more likely to view recycling as a desirable activity. But if the extractor maximizes profit $(\lambda=1)$, it will view the recycler as a rival threatening its market position.

As previously mentioned, we assume that all of the remaining resource stock is extracted in the second period, which precludes the extractor from reducing its output below $s$. If the extractor did reduce its output, it would sell the non-cooperative quantity $q^{N}$ of the Nash equilibrium outcome resulting from competition against the recycler with simultaneous move. To ensure that this will not happen, we explicitly state the following assumption:

$(A 4) s<q^{N}$.

The first step is to derive the recycler's reaction function $r^{*}(q)$ that maximizes $\pi(q, r)$ with respect to $r$. Given $q$, the recycler will enter the market if $\pi\left(q, r^{*}(q)\right)$ is positive; otherwise, the recycler earns zero profit and stays out of the market. If the recycler enters the market, then $r^{*}(q)$ is the interior solution denoted by $r(q)$ to

$$
\pi_{r}(q, r)=0
$$


We show in Appendix 3 that assumptions $(A 1)$ and $(A 2)$ ensure the existence of $r(q)$.

We define $\widetilde{q}$ as the minimum threshold of initial extraction above which recycling becomes profitable, so that $\pi(\widetilde{q}, r(\widetilde{q}))=0$. Observing $\widetilde{q}$, the recycler is indifferent between staying out and entering. For all $q$ higher than $\widetilde{q}$, we have $\pi(q, r(q))>0$ and the recycler enters the market. In contrast, for all $q$ lower than $\widetilde{q}$, we have $\pi(q, r(q))<0$, and hence the recycler is better off securing zero profit by staying out of the market. Therefore, the recycler's reaction function is discontinuous at $\widetilde{q}$, which increases with $F$. If $F$ is so high that $\widetilde{q}=s$, the recycler cannot even make a profit as a monopolist in the second period, and then its reaction function is simply $r^{*}(q)=0$ for all $q \in[0, s]$.

Lemma 2: Under assumptions $(A 1),(A 2)$ and $(A 3)$, the recycler has the following reaction function

$$
r^{*}(q)=\left\{\begin{array}{c}
r(q) \text { when } \widetilde{q} \leq q \leq s, \\
0 \text { otherwise }
\end{array}\right.
$$

with a slope such that $0<r^{\prime}(q)<1$.

Proof: The mathematical techniques of the proof are similar to those used for Lemma 1 and so it is left to the reader to refer to them.

The extractor chooses $q$ to maximize the reduced-form function $\mathbf{V}(q)=V\left(q, r^{*}(q)\right)$, taking into account the possibility and consequences of the recycler's entry. Then, we have

$$
\mathbf{V}(q)=\left\{\begin{array}{c}
V(q, r(q)) \text { if } \widetilde{q} \leq q \leq s, \\
V(q, 0) \text { otherwise. }
\end{array}\right.
$$

A key feature of the general framework is that $\mathbf{V}(q)$ is not concave in $q$ because of the discontinuity in $r^{*}(q)$. More precisely, $\mathbf{V}(q)$ is piecewise concave and discontinuous with a jump at $\widetilde{q}$. Moreover, the jump of $\mathbf{V}(q)$ will be downward or upward depending on the extractor's objective function. Indeed, we have

$$
V_{r}(q, r)=(1-\lambda) \delta p_{2}-\lambda \delta \frac{p_{2}}{\eta_{2}} .
$$

Thus, $V_{r}(q, r)$ is positive when $\lambda=0$, which implies that $\mathbf{V}(q)$ has an upward jump at $\widetilde{q}$. In contrast, when $\lambda=1$, then $V_{r}(q, r)$ is negative, which implies that $\mathbf{V}(q)$ has a downward jump at $\widetilde{q}$.

These technical difficulties make it complicated to address the issue of the recycler's entry. As a first consequence, $\mathbf{V}(q)$ may have two local maxima, of which the one within the range $[\widetilde{q}, s]$ allows the recycler's entry and the one within the range $[0, \widetilde{q}]$ does not. Depending on the size of $F$ which determines the position of $\widetilde{q}$, there is a rich set of entry possibilities that follow Bain's classification (1956, pp. 21-22). If $F$ is so high that $\widetilde{q}>s$, then the recycler stays out for all $q \in[0, s]$. In that case, $\mathbf{V}(q)=V(q, 0)$ has a global maximum at $q_{0}$, that is, the first-period optimal extraction in the absence of recycling. In Bain's terminology, the recycler's entry is blockaded at $q_{0}$, where the extractor behaves as if recycling were irrelevant. 
Let us now turn to the case where $F$ is so small that $\widetilde{q}<s$. Among all the putative equilibrium outcomes, the most interesting ones involve recycling. Suppose for the moment that $q^{*}$ is chosen within the range $[\widetilde{q}, s]$. Restricting the maximization problem to this domain, we can write the Kuhn-Tucker conditions as

$$
\begin{aligned}
V_{q}\left(q, r^{*}(q)\right)+V_{r}\left(q, r^{*}(q)\right) r^{* \prime}(q) & =-\mu\left[\pi_{q}\left(q, r^{*}(q)\right)+\pi_{r}\left(q, r^{*}(q)\right) r^{* \prime}(q)\right], \\
\mu & \geq 0\left(\mu=0 \text { if } \pi\left(q, r^{*}(q)\right)>0\right) .
\end{aligned}
$$

Using these conditions, we distinguish between the equilibrium outcomes, depending on whether the break-even constraint is binding or not.

The case $\mu^{*}=0$.- Assume first that the break-even constraint is not binding in equilibrium, so that $\mu^{*}=0$, which can only be achieved if $q^{*}>\widetilde{q}$. In that case, the recycler enters the market and, given $q^{*}$, responds with $r^{*}\left(q^{*}\right)=r\left(q^{*}\right)$. Taking the recycler's entry for granted, the extractor accommodates recycling with $q_{a}=\arg \max _{q \in[0, s]} V(q, r(q))$ satisfying

$$
V_{q}\left(q, r^{*}(q)\right)=-V_{r}\left(q, r^{*}(q)\right) r^{* \prime}(q)
$$

provided that $F$ is small enough to ensure that $\widetilde{q}<q_{a}$. The left-hand side of (19) is the marginal value obtained from extracting the virgin resource prior to recycling. It captures the standard trade-off between the extractor's returns from present and postponed extractions. The right-hand side of (19) represents the additional value obtained by the extractor from recycling as an opportunity cost of extracting the virgin resource today. This is a strategic effect measuring to what extent recycling affects the return from extraction in the second period.

Introducing (16) into the right-hand side of (19), we get $V_{q}\left(q, r^{*}(q)\right)=\left(-(1-\lambda) \delta p_{2}+\lambda \delta \frac{p_{2}}{\eta_{2}^{*}}\right) r^{* \prime}\left(q_{a}\right)$. We observe that the extractor's incentive to accommodate the recycler's entry with $q_{a}$ depends on whether the extractor is socially benevolent or self-interested. In the case where $\lambda=0$, each unit of resource extracted in the first period improves the second-period welfare by $\delta p_{2} r^{* \prime}\left(q_{a}\right)$ through recycling since $r^{\prime}(q)>0$. In the case where $\lambda=1$, each unit of resource extracted in the first period reduces the second-period marginal revenue by $\delta \frac{p_{2}}{\eta_{2}^{*}} r^{* \prime}\left(q_{a}\right)$ through recycling.

Lemma 3: Suppose that the extractor accommodates the recycler's entry. Then, recycling:

(1) boosts the initial extraction when the extractor maximizes welfare, and

(2) curbs the initial extraction when the extractor maximizes profit.

By choosing extraction in the first period, the extractor is in a position to send a signal to the recycler on the profitability of the market in the second period. The extractor exploits this leadership in two different ways, depending on its objective.

If the extractor maximizes social welfare $(\lambda=0)$, it views recycling as a desirable activity because recycling creates a valuable expansion of the stock of the resource. This is similar to 
what André and Cerdá (2006) call the "scale effect" when they point out that recycling may alleviate resource scarcity.

In contrast, if the extractor maximizes profit $(\lambda=1)$, it views the recycler as a rival that threatens its market position. The prospective entry of the recycler provides the extractor with an incentive to restrict the initial extraction and instead flood the market in the second period, which finally triggers a less aggressive reaction from the recycler.

The case $\mu^{*}>0$.-Assume now that the break-even constraint is binding in equilibrium, i. e., $\mu^{*}>0$, which can only happen at $q^{*}=\widetilde{q}$, where $\mathbf{V}(q)$ is discontinuous. This requires that $F$ be higher than in the case where $\mu^{*}=0$, so that we have $q_{a}<\widetilde{q}<s$. Setting $\widetilde{q}$ triggers the reaction $r^{*}(\widetilde{q}) \in\{0, r(\widetilde{q})\}$ from the recycler, meaning that the extractor may either prevent recycling or allow it to occur.

In the outcome with no recycling, $\widetilde{q}$ is the best choice for the extractor only if $\mathbf{V}(\widetilde{q})=$ $V(\widetilde{q}, 0)>V(\widetilde{q}, r(\widetilde{q}))$. Two requirements are needed for this: $(i) \mathbf{V}(q)$ has a downward jump at $\widetilde{q}$, hence $\lambda=1$, and $(i i)$ the recycler chooses to stay out when it is indifferent between entering and staying out, hence $r^{*}(\widetilde{q})=0$. We will assume this to be the case when $\lambda=1 .^{11}$ Then, $\mathbf{V}(q)$ achieves a unique maximum within the range $[0, \widetilde{q}]$ since $q_{a}<\widetilde{q}$. Finally, the maximum is at $\widetilde{q}$ provided that $\widetilde{q}<q_{0}$. In Bain's terminology, the extractor deters entry by decreasing the initial extraction below the level $q_{0}$ that would be optimally extracted in the absence of recycling. Thus, setting $\widetilde{q}$ to impede the recycler's entry proves costly for the profit-maximizing extractor. But this strategy signals to the recycler the extractor's intention to flood the market and make recycling worthless.

Alternatively, the choice of $\widetilde{q}$ by the extractor may also allow the recycler's entry in equilibrium, under the binding constraint of zero profit. For this to occur, we need that $\mathbf{V}(\widetilde{q})=V(\widetilde{q}, r(\widetilde{q}))>V(\widetilde{q}, 0)$, which requires the following conditions to be met: $(i) \mathbf{V}(q)$ has an upward jump at $\widetilde{q}$, hence $\lambda=0$, and (ii) the recycler chooses to enter when it is indifferent between entering and staying out, hence $r^{*}(\widetilde{q})=r(\widetilde{q})$. We will assume this to be the case when $\lambda=0 .{ }^{12}$ Then, $\mathbf{V}(q)$ achieves a local maximum at $\widetilde{q}$ above $q_{a}$ that would be optimally extracted if the break-even constraint were not binding. Thus, setting $\widetilde{q}$ to encourage entry proves costly for the benevolent extractor. However, this strategy signals to the recycler the extractor's intention to make recycling worthwhile by relaxing the pressure on the price in the second period. We will say that the extractor promotes recycling with $q^{*}=\widetilde{q}$ to distinguish this equilibrium strategy from the accommodating strategy $q_{a}$.

When the benevolent extractor promotes recycling with $\widetilde{q}$, (17) can be rewritten as

$$
V_{q}(q, r(q))=-\delta p_{2} r^{\prime}(q)-\mu \pi_{q}(q, r(q))
$$

by using (16) and the envelope theorem. Furthermore, we know that $\pi_{q}(q, r)=-P_{2}^{\prime}()$.

\footnotetext{
${ }^{11}$ In other terms, in the case $\lambda=1$, we will restrict attention to the pure strategy in which the recycler chooses to stay out with probability 1 when the initial extraction is $\widetilde{q}$.

${ }^{12}$ In other terms, in the case $\lambda=0$, we will restrict attention to the pure strategy in which the recycler chooses to enter with probability 1 when the initial extraction is $\widetilde{q}$.
} 
and, for $q=\widetilde{q}$, we have $-P_{2}^{\prime}() r.(q)=p_{2}-c^{\prime}(r)=\frac{p_{2}}{\varepsilon}$, provided that $\varepsilon>1$ for an interior solution. Introducing this into (20) yields

$$
V_{q}(q, r(q))=-\delta p_{2} r^{\prime}(q)-\mu \frac{p_{2}}{\varepsilon} .
$$

Each term in the right-hand side of (21) reflects specific earnings foregone by the benevolent extractor when it reduces the input for recycling. As with the accommodating strategy, recycling has a valuable effect on the size of the resource stock (first term). In addition, the extractor needs that the proceeds from sales of the recycled resource exactly offset the costs of recycling. Therefore, when promoting recycling, the benevolent extractor must take into consideration the foregone revenue from recycling (second term).

Lemma 4: In equilibrium, the strategy $\widetilde{q}$ is a costly signal for the extractor. This strategy:

(1) promotes recycling when the extractor maximizes welfare, and

(2) deters recycling when the extractor maximizes profit.

Faced with the prospective entry of the recycler, the extractor must consider different strategies, depending on whether it maximizes social welfare or its own revenue. We will distinguish these cases in Sections 5.1 and 5.2, and present our results in the form of two modified Hotelling rules.

\subsection{The extractor maximizes social welfare $(\lambda=0)$}

The extractor's problem is now to design a socially efficient extraction program guaranteeing the financing of recycling. By socially efficient, we mean an extraction program that maximizes the social surplus from extracting the virgin resource and providing the input for recycling less the true budgetary cost of delegating the task of recycling to a profit-maximizing firm.

When $\lambda=0$, the extractor maximizes $W(q, r)=S_{1}(q)+\delta S_{2}(s-q+r)$ under the breakeven constraint (2). From (17) and (21), the first-order condition for the extractor's problem becomes

$$
p_{1}-\delta p_{2}=-p_{2}\left(\delta r^{\prime}(q)+\frac{\mu}{\varepsilon}\right) .
$$

In the absence of recycling, the extractor would be better off setting $q_{0}$, in accordance with the standard Hotelling rule under perfect competition. Faced with prospective recycling, the extractor finds it worthwhile to increase the initial extraction above $q_{0}$, as shown by the right-hand side of (22). By substituting $\delta=\frac{1}{1+\rho}$ into (22), we obtain that the equilibrium solutions revisits the Hotelling rule as follows:

$$
\frac{p_{2}^{*}-p_{1}^{*}}{p_{1}^{*}}=\rho+\frac{p_{2}^{*}}{p_{1}^{*}}\left(r^{\prime}\left(q^{*}\right)+(1+\rho) \frac{\mu^{*}}{\varepsilon^{*}}\right)
$$

provided that $\varepsilon^{*}>1$ for an interior solution at $q^{*}=\widetilde{q}$. As $r^{\prime}(q)>0$ and $\mu^{*} \geq 0$, we see that $\frac{p_{2}^{*}-p_{1}^{*}}{p_{1}^{*}}>\rho$. Thus, the price of the resource increases faster than the rate of interest. The right-hand side of (23) shows that, besides the foregone interest $\rho$, the opportunity cost of 
postponing extraction includes the dual value of recycling foregone from holding the resource in the ground. Recycling is always valuable because it increases the stock of the resource (first term in the parentheses), but recycling is also valuable when the break-even constraint is binding (second term in the parentheses) because proceeds from recycling are needed to offset the recycling costs. The extractor takes this budgetary requirement into account when it promotes recycling with $q^{*}=\widetilde{q}$, but not when it accommodates recycling with $q^{*}=q_{a}$.

We conclude:

Proposition 2: Assume $(i)$ that the extractor and the recycler are separate entities and (ii) that the extractor maximizes welfare. Under assumptions $(A 1),(A 2),(A 3)$ and $(A 4)$, recycling induces the extractor to accelerate the depletion of the resource by setting $q^{*}$ above $q_{0}$, so that

$$
\frac{p_{2}^{*}-p_{1}^{*}}{p_{1}^{*}}>\rho .
$$

Not surprisingly, this result is closely related to that obtained in the benchmark scenario treated in Section 4. One reason for increasing the initial extraction is to generate enough revenue to cover the costs of recycling. The scale effect of recycling is a further reason for accelerating the resource depletion in the present scenario: increased extraction alleviates resource scarcity by providing more input for recycling.

The framework of linear demand and cost functions.-We now derive explicit solutions for $\left(q^{*}, r^{*}().\right)$ in the specific example, when the extractor maximizes social welfare and the recycler is an independent profit maximizer. The social welfare is given by

$$
W(q, r)=a q-q^{2} / 2+a(s-q+r)-(s-q+r)^{2} / 2 .
$$

In the absence of recycling, the optimal extraction is $q_{0}=\frac{s}{2}$. The recycler chooses $r$ to maximize $\pi(q, r)$, which yields the reaction function

$$
r^{*}(q)=\left\{\begin{array}{c}
r(q)=\frac{1}{2}(a-s-c+q) \text { when } \widetilde{q} \leq q \leq s, \\
0 \text { otherwise }
\end{array}\right.
$$

where

$$
\widetilde{q}=s+c-a+2 \sqrt{F} .
$$

Observe that $\widetilde{q}$ is increasing with $F$, so that $\widetilde{q}>s$ when $F>\bar{F}=\frac{(a-c)^{2}}{4}$. In that case, the fixed cost is so large that the recycler stays out of the market, whatever the initial extraction. Hence, $\bar{F}$ is the maximum level of fixed cost below which the recycler may find the market attractive. To ensure the relevance of recycling, we assume that $F \leq \bar{F}$.

Figure 2 depicts the extractor's isowelfare curves (in black) given by (24) and the recycler's isoprofit curves (in gray) in $(q, r)$ space. As in Figure 1, isowelfare curves are associated with higher levels of welfare when moving from $A$ to $B$, and profit levels are higher as the isoprofit curves shift from $C$ to $D$ along the line $\pi_{r}(q, r)=0$. The segment $[C, D]$ represents that part of the recycler's reaction function for which recycling is profitable, for a given $F$. The extractor's problem is to find a location on $[C, D]$ that maximizes welfare. 


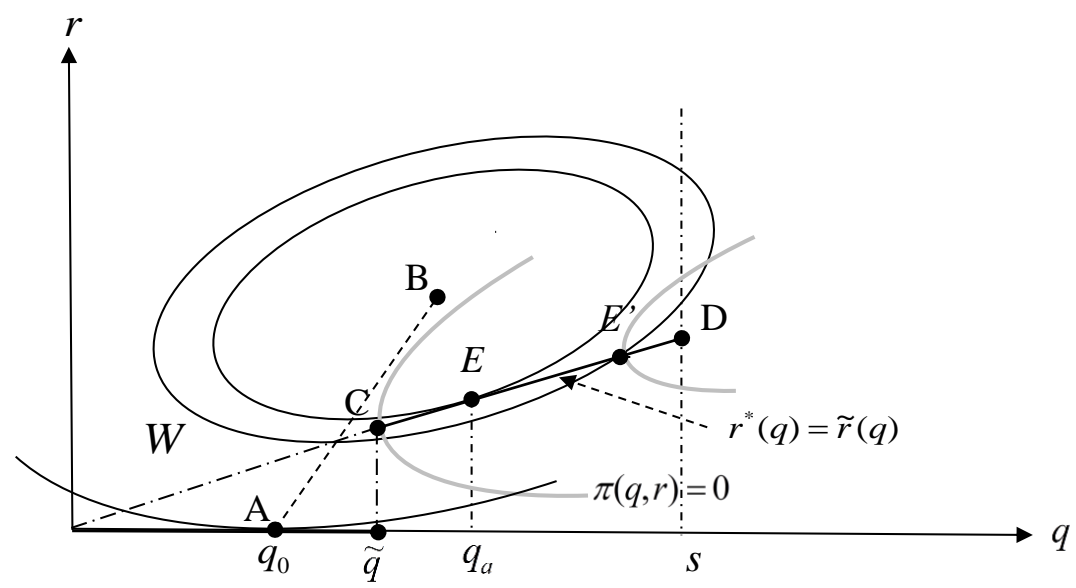

Figure 2: Equilibrium solutions for a benevolent extractor confronted by an independent recycler 
Figure 2 illustrates two different equilibrium outcomes corresponding to points $E$ and $E^{\prime}$, respectively.

At point $E$, whose coordinate on the $q$-axis is $q_{a}$, the isowelfare curve is tangent to $[C, D]$. The highest level of welfare is achieved at $E$ provided that $\widetilde{q} \leq q_{a}$. This requires that $F$ be lower than

$$
F_{a}=\frac{(4 a-3 c-2 s)^{2}}{25} .
$$

In that case, the break-even constraint is not binding, that is, $\mu^{*}=0$, and hence, the extractor accommodates recycling. Calculations made from (22) yield

$$
q_{a}=\frac{3 a-c+s}{5} .
$$

Note that the figure is drawn under the assumption that

$$
W\left(q_{0}, 0\right)<W(s, r(s))
$$

meaning that it would be socially more efficient for the benevolent extractor to recycle the whole stock of the resource rather than to extract the virgin resource without recycling. Throughout this example, we focus on the set of parameters satisfying this assumption for the sake of simplicity.

If $F$ becomes so large that $q_{a} \leq \widetilde{q}$, then the part $r(q)$ of the recycler's reaction function for which entry is profitable shrinks to fit the segment $\left[E^{\prime}, D\right]$ on Figure 2. The best the extractor can do to meet the break-even constraint is to choose the point $E^{\prime}$ on the reaction function whose coordinates are $(\widetilde{q}, r(\widetilde{q}))$. Recall that the recycler chooses to enter with probability 1 when the initial extraction is $\widetilde{q}$ (see Footnote 12). ${ }^{13}$ At $E^{\prime}$, the break-even constraint is binding. Thus, the extractor promotes recycling.

We can state these results as follows.

Corollary 2: Under assumptions (A4) and (29) within the framework of linear demand and cost functions, the best choice for the benevolent extractor is:

(1) To accommodate recycling with $\min \left\{q_{a}, s\right\}$ when $F \leq F_{a}$;

(2) To promote recycling with $\widetilde{q}=s+c-a+2 \sqrt{F}$ when $F_{a}<F<\bar{F}$;

(3) To ignore recycling with $q_{0}=\frac{s}{2}$ when $\bar{F} \leq F$.

Proof: see Appendix 3.

\subsection{The extractor only maximizes its own revenue $(\lambda=1)$}

Assume now that the extractor only serves its own interests; that is, $V(q, r)=R(q, r)=$ $P_{1}(q) q+\delta P_{2}(s-q+r)(s-q)$. Let $M R_{1}(q)=P_{1}(q)+P_{1}^{\prime}(q) q$ and $M R_{2}(q)=P_{2}(s-q+$ $r)+P_{2}^{\prime}(s-q+r)(s-q)$ denote the marginal revenues earned by the extractor in the first and the second period, respectively. By introducing the price elasticity of demand $\eta_{t}$ into these

\footnotetext{
${ }^{13}$ Otherwise, there can be no equilibrium at $E^{\prime}$ so long as the extractor thinks that the recycler stays out with a positive probability.
} 
expressions, we get $M R_{t}(q)=p_{t}\left(1-\frac{1}{\eta_{t}}\right)$ and we will assume that $\eta_{t}^{*}>1$ in equilibrium, so that the marginal revenue is positive in both periods.

The first-period optimal extraction in the absence of recycling is $q_{0}=\arg \max _{q} R(q, 0)$. This monopoly output satisfies the corresponding Hotelling rule that marginal revenues increase at the rate of interest ${ }^{14}$; that is,

$$
\frac{M R_{2}\left(q_{0}\right)-M R_{1}\left(q_{0}\right)}{M R_{1}\left(q_{0}\right)}=\rho .
$$

To examine how recycling affects this rule, we focus on the equilibrium outcome that allows the entry of the recycler. As previously shown, the break-even constraint is not binding in this case, hence $\mu^{*}=0$. The extractor accommodates recycling with $q_{a}$ satisfying (19) and the recycler responds to the initial extraction with $r^{*}\left(q_{a}\right)=r\left(q_{a}\right)$. From Lemma 3, we know that recycling induces the extractor to curb the initial extraction.

The condition (19) can be rewritten as

$$
M R_{1}(q)-\delta M R_{2}(q)=\delta \frac{p_{2}}{\eta_{2}} r^{\prime}(q)
$$

Substituting $\delta=\frac{1}{1+\rho}$ into (31) yields

$$
\frac{M R_{2}(q)-M R_{1}(q)}{M R_{1}(q)}=\rho-\frac{p_{2}}{p_{1}} \frac{\eta_{1}}{\left(\eta_{1}-1\right) \eta_{2}} r^{\prime}(q) .
$$

Equation (32) says that the rate of increase in the extractor's marginal revenue must equal the opportunity cost of deferred extraction. As usual, this cost is made up of the foregone interest, $\rho$. The novel insight here is the second term in the right-hand side of (32), which can be interpreted as capturing the savings in costs due to softening competition from the recycler. As a result, the extractor accommodates recycling so that its marginal revenue rises more slowly than the rate of interest. Extracting more of the resource prior to recycling in accordance with the standard Hotelling rule would weaken the market position of the extractor in the next period by making the recycler more aggressive.

Proposition 3: Assume ( $i$ ) that the extractor and the recycler are separate entities, (ii) that the extractor accommodates recycling to maximize its own revenue. Under assumptions $(A 1),(A 2),(A 3)$ and $(A 4)$, recycling induces the extractor to slow down the depletion of the resource by setting $q_{a}$ below $q_{0}$, so that

$$
\frac{M R_{2}\left(q_{a}\right)-M R_{1}\left(q_{a}\right)}{M R_{1}\left(q_{a}\right)}<\rho .
$$

As pointed out by Hotelling (1931), the resource is extracted less rapidly by a monopoly than by competitive producers because of "the general tendency for production to be retarded under monopoly". The theoretical literature has since shown that this result is fairly robust

\footnotetext{
${ }^{14}$ See Stiglitz (1976), for instance.
} 
(see, for instance, Stiglitz 1976; Peterson and Fisher 1977). From Lemma 4, we know that the monopolist deters the entry of the recycler by setting $\widetilde{q}$ below $q_{0}$. The reduction in extraction signals to the recycler the extractor's intention to flood the market enough to make recycling worthless. Finally, Proposition 3 sheds light on a further motivation for the monopolist to conserve the resource more than predicted by the Hotelling rule: the monopolist defends its market position by mitigating the recycler's reaction.

By rearranging Eq. (32), we obtain

$$
\frac{p_{2}-p_{1}}{p_{1}}=\frac{\left(\eta_{1}-1\right) \eta_{2}}{\eta_{1}\left(\eta_{2}-1\right)} \rho-\frac{\eta_{2}-\eta_{1}}{\eta_{1}\left(\eta_{2}-1\right)}-\frac{p_{2}}{p_{1}} \frac{r^{\prime}(q)}{\eta_{2}-1} .
$$

We can see that whether prices will grow or fall depends on two factors: $(i)$ the change in the elasticity of demand from one period to the next; and (ii) the severity of the recycling reaction measured by the slope of $r(q)$. Assume that the elasticity of demand increases over time due, for instance, to the discovery of substitutes for the resource: $\eta_{2}>\eta_{1}>1$. Then, the second and third terms in the right-hand side of (33) are negative and the weight given to the rate of interest in the first term is lower than 1 . It follows that $\frac{p_{2}-p_{1}}{p_{1}}<\rho$. This is consistent with standard predictions that monopoly power tends to flatten the price path of the resource (Stiglitz 1976; Gaudet 2007). The third term in the right-hand side of (33) shows that the extractor's internalization of the recycler's reaction tends to further flatten the price path. The addition of the three negative effects might explain falling prices for some exhaustible resources, as revealed by casual inspection of price data (Slade and Thille 2009).

The framework of linear demand and cost functions. - We now derive explicit solutions $\left(q^{*}, r^{*}().\right)$ in the specific example where the extractor and the recycler are separate entities which both maximize profit.

In Section 5.1, we saw that $r^{*}(q)$ is given by $(25)$. The extractor's objective function is now

$$
R(q, r)=(a-q) q+(a-s+q-r)(s-q) .
$$

We already know that $\widetilde{q}>s$ provided that $F>\bar{F}=\frac{(a-c)^{2}}{4}$. In that case, the fixed cost is so large for the recycler that entry is never profitable. Consequently, the extractor can ignore recycling altogether and exercise unrestrained monopoly power by setting $q_{0}=$ $\arg \max _{q} R(q, 0)=\frac{s}{2}$.

Assume now that $F \leq \bar{F}$, so that the recycler will find the market attractive for all $q \in[\widetilde{q}, s]$. Figure 3 depicts the extractor's isoprofit curves (in gray) given by (34) and the recycling reaction $(25)$ in $(q, r)$ space. The isoprofit curves are hyperbolae of the form $\{(q, r): R(q, r)=\mathrm{R}\}$ for some isoprofit level $\mathrm{R}$. The segment $[A, B]$ cuts each of the isoprofit curves at its maximum. In particular, given $r=0, R(q, r)$ is maximized at the point $A$ whose coordinates are $\left(q_{0}, 0\right)$ yielding the monopoly outcome for the extractor. The profit levels decrease moving away from $A$ to $B$. The isoprofit curve is tangent to $[C, D]$ at $E$, and 


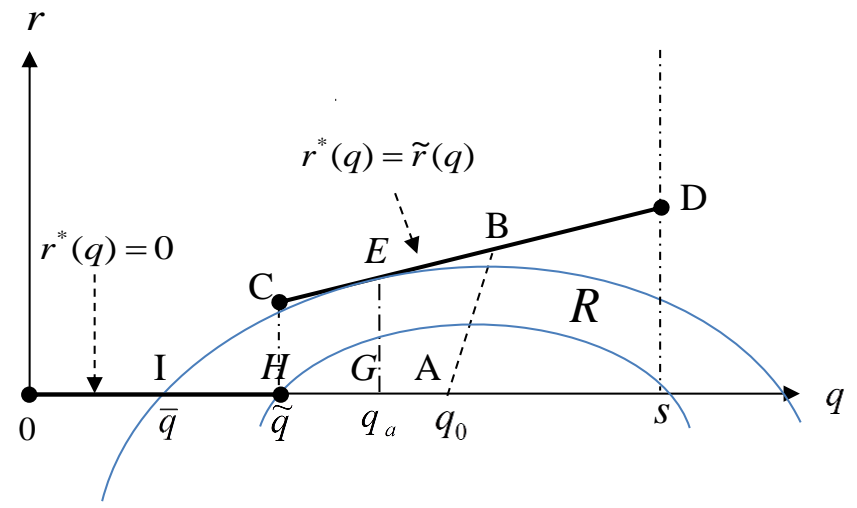

Figure 3: Equilibrium solutions for a self-interested extractor confronted by an independent recycler 
this curve meets the $q$-axis at that $I$ whose coordinates are $(\bar{q}, 0)$.

Figure 3 illustrates the case where $H$ lies between $I$ and $G$, whose coordinates are $\left(q_{a}, 0\right)$. In that case, the extractor maximizes its revenue at $H$ by setting the initial extraction to be $\widetilde{q}$, which deters the recycler from entering the market. Recall that the recycler chooses to stay out with probability 1 when the initial extraction is $\widetilde{q}$ (see Footnote 11). ${ }^{15}$ The figure is drawn under the assumption

$$
R\left(q_{0}, 0\right)>R\left(q_{a}, r\left(q_{a}\right)\right) .
$$

meaning that it would be more profitable for the self-interested extractor to ignore recycling rather than to accommodate it. Throughout this example, we work under this assumption to simplify matters.

Suppose that the fixed cost is so low that $\widetilde{q}<\bar{q}$ and $H$ lies to the left of $I$. This case occurs when $F<F_{A}$, where

$F_{A}=\frac{1}{192}\left[47(a-c)^{2}+8 s(s-5(a-c))+4 \sqrt{3}(2(a-c)-s) \sqrt{4 s(2(a-c)-s)-(a-c)^{2}}\right]$.

Then, the best choice for the extractor is to accommodate the recycler at point $E$ by setting the initial extraction at $q_{a}$. Computations based on (31) yield

$$
q_{a}=\frac{2 s+a-c}{6}
$$

Observe that $q_{a}<q_{0}$ under the assumption (3), which is consistent with the result stated in Proposition 3: to accommodate the recycler's entry, the extractor must reduce the initial extraction below the output level that would prevail in the absence of recycling.

In contrast, if $F$ is so high that $q_{0}<\widetilde{q}$ and $H$ lies to the right of $A$, then the extractor prefers choosing $q_{0}$ to $\widetilde{q}$, thereby blockading the recycler's entry. Such is the case when $F>F_{d}$, where

$$
F_{d}=\frac{(2(a-c)-s)^{2}}{16} .
$$

We can summarize the previous discussion as follows.

Corollary 3: Under assumptions (A4) and (35) within the framework of linear demand and cost functions, the best choice for the self-interested extractor is:

(1) To accommodate recycling with $q_{a}=\frac{2 s+a-c}{6}$ when $F<F_{A}$,

(2) To deter recycling with $\widetilde{q}=s+c-a+2 \sqrt{F}$ when $F_{A} \leq F<F_{d}$;

(3) To blockade recycling with $q_{0}=\frac{s}{2}$ when $F_{d} \leq F \leq \bar{F}$.

Proof: see Appendix 4.

\footnotetext{
${ }^{15}$ Otherwise, there can be no equilibrium at $H$ so long as the extractor thinks that there is a positive probability of entry.
} 


\section{Conclusion}

In this paper, we have examined the strategic behavior of the extractor of an exhaustible resource in the face of prospective recycling. For this, we have investigated a two-period model. Extraction of the virgin resource in the first period provides the input for recycling in the second period, which is when the market is shared between the extractor and the recycler. Hence, the first-period choice of extraction signals whether and to what extent recycling is profitable in the second period.

The intuitions underlying the equilibrium outcomes differ according to the objective pursued by the extractor. When the extractor seeks to maximize social welfare, it views recycling as an opportunity to increase the resource stock beyond its natural limits. In contrast, the profit-maximizing extractor sees recycling as a threat to its market position. Therefore, the socially benevolent extractor is more willing to encourage recycling than the self-interested extractor. Our findings shed fresh light on the modifications introduced in the Hotelling rule by recycling in each equilibrium outcome.

In the case where the extractor maximizes welfare, we find that it must accelerate the resource depletion to accommodate recycling when fixed costs are low. As fixed costs rise above some threshold level, the extractor still more accelerates the resource depletion, accepting a costly increase in output to promote recycling.

In the case where the extractor maximizes profit, it must slow down the resource depletion to send a signal to the recycler about the market's profitability. When fixed costs of recycling are low, the signal indicates that the extractor accommodates recycling with the intention of softening the blow of recycling. In contrast, when fixed costs are higher, the extractor signals to the recycler that it will make the recycling business unattractive. The decrease in extraction is fine tuned to exert just enough pressure on the market price to deter recycling.

Given that the model with sequential moves investigated here is very simple, a natural extension would be to switch to an infinite horizon. One possible approach in this direction is to assume that the extractor and the recycler use Markovian strategies in an alternatingmove infinite-horizon model in the spirit of Maskin and Tirole (1987). According to them, the switch to a long-run model built on the infinite repetition of a short-run model with sequential moves yields equilibrium outcomes that are more competitive than those obtained in the short-run model. 


\section{Appendix}

\subsection{Appendix 1: Proof of Lemma 1}

In the first step, we show that the solution $\widehat{r}(q, \mu)$ of $W_{r}^{n}(q, r)+\mu \pi_{r}(q, r)=0$ is a local maximum.

Let the Lagrangian be $L(q, r)=W^{n}(q, r)+\mu\left[P_{2}(s-q+r) r-c(r)-F\right]$. If $L_{r}(q, r)=0$ has an interior solution $\widehat{r}(q, \mu)$, then we have

$$
\widehat{r}(q, \mu)=-\frac{\mu+\delta}{\mu} \frac{P_{2}(s-q+r)-c^{\prime}(r)}{P_{2}^{\prime}(s-q+r)} .
$$

We now check that the second-order condition for $\widehat{r}(q, \mu)$ to be a local maximum of $L(q, r)$ is satisfied. By substituting $\widehat{r}(q, \mu)$ for $r$ in

$$
L_{r r}(q, r)=\delta\left[P_{2}^{\prime}(s-q+r)-c^{\prime \prime}(r)\right]+\mu\left[2 P_{2}^{\prime}(s-q+r)+P_{2}^{\prime \prime}(s-q+r) r-c^{\prime \prime}(r)\right],
$$

we obtain

$$
\begin{aligned}
& L_{r r}(q, \widehat{r}(q, \mu)) \\
= & (\delta+\mu)\left[P_{2}^{\prime}(s-q+\widehat{r}(q, \mu))-c^{\prime \prime}(\widehat{r}(q, \mu))-\frac{P_{2}(s-q+\widehat{r}(q, \mu))-c^{\prime}(\widehat{r}(q, \mu))}{P_{2}^{\prime}(s-q+\widehat{r}(q, \mu))} P_{2}^{\prime \prime}(s-q+\widehat{r}(q, \mu))\right] \\
& +\mu P_{2}^{\prime}(s-q+\widehat{r}(q, \mu)) .
\end{aligned}
$$

Given that $P_{2}($.$) is log-concave by assumption (A 2)$, we know that $P_{2}^{\prime \prime}()<.\frac{P_{2}^{\prime}()^{2}}{P_{2}(.)}$. Thus, we have

$$
L_{r r}(q, \widehat{r}(q, \mu))<\mu P_{2}^{\prime}(.)-(\delta+\mu) c^{\prime \prime}(.)+\frac{(\delta+\mu) c^{\prime}(.)}{P_{2}(.)} P_{2}^{\prime}(.) .
$$

Under assumption $(A 1)$, the right-hand side of $(42)$ is negative, and so $\widehat{r}(q, \mu)$ is a local maximum.

In the second step, we show that $0<\widehat{r}_{q}(q, \mu)<1$.

Differentiating $L_{r}(q, \widehat{r}(q, \mu))=0$ yields the slope of $\widehat{r}_{q}(q, \mu)$ with respect to $q$ :

$$
\widehat{r}_{q}(q, \mu)=-\frac{L_{r q}}{L_{r r}},=\frac{\delta P_{2}^{\prime}(.)+\mu\left[P_{2}^{\prime}(.)+P_{2}^{\prime \prime}(.) r\right]}{\delta\left[P_{2}^{\prime}(.)-c^{\prime \prime}(.)\right]+\mu\left[2 P_{2}^{\prime}(.)+P_{2}^{\prime \prime}(.) r-c^{\prime \prime}(.)\right]} .
$$

where $-L_{r q}=\delta P_{2}^{\prime}()+.\mu\left[P_{2}^{\prime}()+.P_{2}^{\prime \prime}() r.\right]$. As $L_{r r}<0$, we have sign $\left(\widehat{r}_{q}\right)=\operatorname{sign}\left(L_{r q}\right)$. After substitution of $\widehat{r}(q, \mu)$ for $r$, we obtain

$$
-L_{r q}=(\delta+\mu)\left[P_{2}^{\prime}(.)-\left(P_{2}(.)-c^{\prime}(.)\right) P_{2}^{\prime \prime}(.) / P_{2}^{\prime}(.)\right] .
$$

Again using the log-concavity of $P_{2}($.$) , we have$

$$
-L_{r q}<(\delta+\mu)\left[P_{2}^{\prime}(.)-\left(P_{2}(.)-c^{\prime}(.)\right) \frac{P_{2}^{\prime}(.)}{P_{2}(.)}\right] .
$$


The right-hand side of $(45)$ reduces to $(\delta+\mu) c^{\prime}(.) \frac{P_{2}^{\prime}(.)}{P_{2}(.)}<0$. It follows that $-L_{r q}<0$ and, therefore, $\widehat{r}_{q}(q, \mu)>0$.

Further calculations yield

$$
\widehat{r}_{q}(q, \mu)-1=\frac{\delta c^{\prime \prime}(.)+\mu\left[-P_{2}^{\prime}(.)+c^{\prime \prime}(.)\right]}{L_{r r}} .
$$

The numerator of the fraction given on the right-hand side of (46) is strictly positive, whereas the denominator $L_{r r}$ is negative. We can conclude that $\widehat{r}_{q}(q, \mu)-1<0$.

In the third step, we show that $\widehat{r}(q, \mu)$ is an interior solution under assumption $(A 3)$, which falls short of $q$.

Given that recycling cannot emerge ex nihilo, a minimum amount $\underline{q}>0$ of the virgin resource is needed to produce the first unit of recycled output; that is, $\widehat{r}(q, \mu)=0$. As $\underline{q}>0$, we have $\widehat{r}(0, \mu)<\widehat{r}(\underline{q}, \mu)=0$ since $\widehat{r}(q, \mu)$ is upward sloping with respect to $q$. This inequality is equivalent to $L_{r}(0,0)=-(\mu+\delta)\left[P_{2}(s)-c^{\prime}(0)\right] \leq 0$, which holds under assumption $(A 3)$. Moreover, $L_{r}(s, 0)=(\mu+\delta)\left[P_{2}(0)-c^{\prime}(0)\right]$ is positive under assumption (A3). Thus, $\widehat{r}(\underline{q}, \mu)=0<\widehat{r}(s, \mu)$, and so $\underline{q}<s$. Finally, for all $q$ higher than $\underline{q}$, we have $\widehat{r}(q, \mu)-q<\widehat{r}(\underline{q}, \mu)-\underline{q}$ since $\widehat{r}_{q}(q, \mu)-1<0$, and thus $\widehat{r}(q, \mu)<q$ : the virgin resource is never fully recycled.

\subsection{Appendix 2: Proof of Proposition 1}

The social planner maximizes $W^{n}(q, r)$ under the break-even constraint (2). Assuming an interior solution, the first-order conditions for this problem are

$$
\begin{aligned}
P_{1}(q)-\delta P_{2}(s-q+r) & =\mu P_{2}^{\prime}(s-q+r) r, \\
P_{2}(s-q+r)-c^{\prime}(r) & =-\frac{\mu}{(\delta+\mu)} P_{2}^{\prime}(s-q+r) r, \\
\mu\left[P_{2}(s-q+r) r-c(r)-F\right] & =0 .
\end{aligned}
$$

If $\mu=0$, (48) immediately shows that the recycling output must be sold at marginal cost. If $\mu$ is positive, then the constraint (2) is binding and the social planner must use the proceeds from sales of the recycled product to finance the recycling costs. Using $\varepsilon$, the first-order condition (48) yields the standard Ramsey formula

$$
\frac{P_{2}(s-q+r)-c^{\prime}(r)}{P_{2}(s-q+r)}=\frac{\mu}{(\delta+\mu)} \frac{1}{\varepsilon} .
$$

Note that (50) can be rewritten as follows:

$$
p_{2}\left(1-\frac{\mu}{(\delta+\mu) \varepsilon}\right)=c^{\prime}(r) \text {. }
$$

Thus, we must have $\varepsilon>\frac{\mu}{\delta+\mu}$ for an interior solution. If this were not the case, the left-hand side of (51) would be negative and, hence, could not be equal to marginal cost. 
Furthermore, setting $\varepsilon$ in (47) yields:

$$
\frac{p_{2}}{p_{1}}=\frac{1}{\delta}+\frac{\mu}{\delta \varepsilon} \frac{p_{2}}{p_{1}}
$$

By substituting $\delta=\frac{1}{1+\rho}$ into (52), we obtain the variant of the Hotelling rule(8). Moreover, we can write $(52)$ as

$$
\frac{p_{2}}{p_{1}}\left(1-\frac{\mu}{\delta \varepsilon}\right)=\frac{1}{\delta}
$$

which shows that $\varepsilon$ must be higher than $\frac{\mu}{\delta}$ to get an interior solution. If so, then the condition $\varepsilon>\frac{\mu}{\delta+\mu}$ needed for the existence of an interior solution of Equation (51) is also satisfied.

\subsection{Appendix 3: Proof of Corollary 2}

With linear demand and cost functions, assumption $(A 4)$ can be written as ${ }^{16}$

$$
s<\frac{a+c}{3} .
$$

By substituting $r^{*}(q)$ for $r$ in (24), we obtain the reduced-form function

$$
\mathbf{V}(q)=\left\{\begin{array}{c}
a q-q^{2} / 2+a(a-c+s-q) / 2-(a-c+s-q)^{2} / 8 \text { if } \widetilde{q} \leq q \leq s, \\
-q^{2}+s q+a s-s^{2} / 2 \text { otherwise. }
\end{array}\right.
$$

Assumption (29) boils down to $W\left(q_{0}, 0\right)<\mathbf{V}(s)$, which requires that $s$ belongs to the interval $\left(a-c, \sqrt{\frac{(a-c)(3 a+c)}{2}}\right)$.

Substituting (28) into (25) yields

$$
r\left(q_{a}\right)=\frac{4 a-3 c-2 s}{5} .
$$

From (28), we see that $q_{a} \leq s$ provided that $c \geq 3 a-4 s$. Otherwise, we have $\widetilde{q} \leq s<q_{a}$ when $F \leq \bar{F}$, and thus $\mathbf{V}(q)$ achieves a global maximum at $s$ from (29). Moreover, $r\left(q_{a}\right) \geq 0$ requires that $c \leq \frac{2}{3}(2 a-s)$, which holds under (29). It turns out that $F_{a}<\bar{F}$ when $c \geq 3 a-4 s$ holds. One can also check that, under assumption $(A 4)$ and $c<a$, we have $q_{0}<q_{a}$.

There are three possibilities:

(i) $\widetilde{q}<q_{0}<q_{a}<s$. The inequality $\widetilde{q}<q_{0}$ requires that $F<F_{d}$, where $F_{d}=\frac{(2(a-c)-s)^{2}}{16}$. Under (29), we have $F_{d}<F_{a}$ because this requires that $c<\frac{26 a-13 s}{22}$ and we have $\sqrt{\frac{(a-c)(3 a+c)}{2}}<\frac{26 a-13 s}{22}$. Then, $\mathbf{V}(q)$ achieves a unique maximum at $q_{a}$.

\footnotetext{
${ }^{16}$ Let $p_{2}^{N}$ and $r^{N}$ respectively denote the market clearing price and the recycler's output in the second period Nash equilibrium with no resource exaustion that would result from competition between the extractor and the recycler. Within this specific framework, this equilibrium outcome is characterized by $q^{N}=\frac{a+c}{3}, r^{N}=\frac{a-2 c}{3}$ and $p_{2}^{N}=\frac{a+c}{3}$.
} 
(ii) $q_{0} \leq \widetilde{q} \leq q_{a}<s$. This requires that $F_{d} \leq F \leq F_{a}$. Under (29), $\mathbf{V}(q)$ achieves a global maximum at $q_{a}$ because $W\left(q_{0}, 0\right)<\mathbf{V}(s)<\mathbf{V}\left(q_{a}\right)$.

(iii) $q_{0} \leq q_{a}<\widetilde{q}<s$. This requires that $F_{a}<F<\bar{F}$. Under (29), $\mathbf{V}(q)$ achieves a global maximum at $\widetilde{q}$ because $W\left(q_{0}, 0\right)<\mathbf{V}(s)<\mathbf{V}(\widetilde{q})$.

\subsection{Appendix 4: Proof of Corollary 3}

By substituting $r^{*}(q)$ for $r$ in (34) within the framework of linear demand and cost functions, we obtain the reduced-form function

$$
\mathbf{V}(q)=\left\{\begin{array}{c}
(a-q) q+(a-s+q+c)(s-q) / 2 \text { if } \widetilde{q} \leq q \leq s \\
(a-q) q+(a-s+q)(s-q) \text { otherwise }
\end{array}\right.
$$

Assumption (35) requires that $s$ belongs to the interval $\left(a-c, \frac{2+\sqrt{3}}{2}(a-c)\right)$ and assumption $(A 4)$ requires that $s<\frac{a+c}{3}$. Under these assumptions, the fixed costs thresholds are ranked in the following order

$$
F_{A}<F_{d}<\bar{F} \text {. }
$$

By solving the equation $R(q, 0)=R\left(q_{a}, r\left(q_{a}\right)\right)$ for $q$, we obtain an explicit formula for $\bar{q}$ :

$$
\bar{q}=\frac{s}{2}-\frac{\sqrt{3}}{12} \sqrt{4 s(2(a-c)-s)-(a-c)^{2}} .
$$

One can easily check that $4 s(2(a-c)-s)-(a-c)^{2}>0$ for all $s \in\left(a-c, \frac{2+\sqrt{3}}{2}(a-c)\right)$. Thus, $\bar{q}$ does exist for all $s<\frac{2+\sqrt{3}}{2}(a-c)$, and we have $\bar{q}<q_{0}$. Further calculations show that $\widetilde{q}>\bar{q}$ holds only if $F \geq F_{A}$.

Depending on the level of the recycler's fixed costs, which determines the location of $\widetilde{q}$, there are three possibilities:

(i) $\widetilde{q}<\bar{q}<q_{0}$. In the case where $\widetilde{q}<\bar{q}$, fixed costs are so small that $F<F_{A}$. Then, $\mathbf{V}(q)$ has two local maxima at $\widetilde{q}$ and $q_{a}$, with a global maximum at $q_{a}$ because $\widetilde{q}<\bar{q}$ implies that $R(\widetilde{q}, 0)<R\left(q_{a}, r\left(q_{a}\right)\right)=\mathbf{V}\left(q_{a}\right)$.

(ii) $\bar{q} \leq \widetilde{q}<q_{0}$. In this case, fixed costs are higher, so that $F_{A} \leq F<F_{d}$. As $\bar{q} \leq \widetilde{q}$, we have $R\left(q_{a}, r\left(q_{a}\right)\right) \leq R(\widetilde{q}, 0)$, which rules out $q_{a}$ as a possible equilibrium. Moreover, $\widetilde{q}<q_{0}$ implies that $F<F_{d}$, and one can check that $F_{d}<\bar{F}$ when $s<\frac{2+\sqrt{3}}{2}(a-c)$. Since $\mathbf{V}(q)$ is increasing on $[0, \widetilde{q}], \mathbf{V}(q)$ is maximized at $\widetilde{q}$.

(iii) $q_{0} \leq \widetilde{q} \leq s$. Now, fixed costs are even higher so that $F_{d} \leq F \leq \bar{F}$. As $q_{a}<q_{0}, \mathbf{V}(q)$ is decreasing on $(\widetilde{q}, s]$, therefore $\mathbf{V}(q)$ achieves a unique maximum at $q_{0}$. 


\section{References}

[1] André, F. J., and E. Cerdá. 2006. On the Dynamics of Recycling and Natural Resources, Environmental and Resource Economics, 33, 2, 199-221.

[2] Baksi, S., and N. V. Long. 2009. Endogenous Consumer Participation and the Recycling Problem, Australian Economic Papers, 48, 4, 281-295.

[3] Bain, J.S. Barriers to New Competition. Cambridge, MA: Harvard University Press, 1956.

[4] Baumol, W. J., and D. F. Bradford. 1970. Optimal Departures From Marginal Cost Pricing, American Economic Review, 60, 3, 265-283.

[5] Boiteux, M. 1956. Sur la Gestion des Monopoles Publics Astreints à l'Equilibre Budgétaire, Econometrica, 24, 1, 22-40; translated as On the Management of Public Monopolies Subject to Budgetary Constraints, Journal of Economic Theory, 3 (1971), 219-240.

[6] Cordell, D., J.-O. Drangert, and S. White. 2009. The Story of Phosphorus: Global Food Security and Food for Thought, Global Environmental Change, 19, 292-305

[7] Ensink, J. H. J., T. Mahmood, W. van der Hoek, L. Raschid-Sally, and F. P. Amerasinghe. 2004. A Nationwide Assessment of Wastewater Use in Pakistan: An Obscure Activity or a Vitally Important One? Water Policy, 6, 197-206.

[8] Friedman, M. Price Theory. Aldine Publishing Co., Chicago, 1967.

[9] Gaudet, G., P. Lasserre, and N. Van Long. 1995. Optimal Resource Royalties with Unknown and Temporally Independent Extraction Cost Structures, International Economic Review, 36, 3, 715-749.

[10] Gaudet, G., and N. Van Long. 2003. Recycling Redux: A Nash-Cournot Approach, Japanese Economic Review, 54, 4, 409-419.

[11] Gaudet, G. 2007. Natural Resource Economics under the Rule of Hotelling, Canadian Journal of Economics, 40, 1033-1059.

[12] Gilbert, R., J., and S. M. Goldman. 1978. Potential Competition and the Monopoly Price of an Exhaustible Resource, Journal of Economic Theory,17, 2, 319-331.

[13] Grant, D. 1999. Recycling and Market Power: A More General Model and ReEvaluation of the Evidence, International Journal of Industrial Organization, 17, 59-80.

[14] Hollander, A., and P. Lasserre. 1988. Monopoly and the Pre-Emption of Competitive Recycling, International Journal of Industrial Organization, 6, 489-497. 
[15] Hotelling, H. 1931. The Economics of Exhaustible Resources, Journal of Political Economy, 39, 2, 137-175.

[16] Livingston, D., H. Colebatch, and N. Ashbolt. 2005. Sustainable Water Paradigm Shift: Does Changing Discourse Mean Change in Organisation?, Working Paper, School of Civil and Environmental Engineering, University of New South Wales, Sydney, Australia.

[17] Martin, R. 1982. Monopoly Power and the Recycling of Raw Materials, Journal of Industrial Economics, 30, 4, 405-419.

[18] Maskin, E., and J. Tirole. 1987. A Theory of Dynamic Oligopoly, III, European Economic Review, 31, 947-968.

[19] Peterson, F. M., and A. C. Fisher. 1977. The Exploitation of Extractive Resources: A Survey, The Economic Journal, 87, 348, 681-721

[20] Ramsey, F. P. 1972. A Contribution to the Theory of Taxation, Economic Journal, $37,145,47-61$.

[21] Sartorius, C. 2011. Lead Market Potential for Phosphorus Recycling Technologies in Germany, in "Innovation for Green Growth", Science Press Beijing, 2014, Rongping, M., and R. Meckl (eds), 275-296.

[22] Seyhan, D., H.-P. Weikard, and E. V. Ierland. 2012. An Economic Model of Longterm Phosphorus Extraction and Recycling, Resources, Conservation and Recycling, 61, 103-108.

[23] Slade, M. E., and H. Thille. 2009. Whither Hotelling: Tests of the Theory of Exhaustible Resources, Annual Review of Resource Economics, 1, 239-260.

[24] Stiglitz, J. 1976. Monopoly and the Rate of Extraction of Exhaustible Resources, American Economic Review, 66, 4, 655-661.

[25] Suslow, V.1986. Estimating Monopoly Behavior with Competitive Recycling: An application to Alcoa, RAND Journal of Economics, 17, 3, 389-403.

[26] Swan, P. L. 1980. The Influence of Recycling on Monopoly Power, Journal of Political Economy, 88, 1, 76-99.

[27] Tullock, G. 1979. Monopoly and the Rate of Extraction of Exhaustible Resources, American Economic Review, 69, 1, 231-233.

[28] Weikard, H. P., and D. Seyhan. 2009. Distribution of Phosphorus Resources Between Rich and Poor Countries: The Effect of Recycling, Ecological Economics, 1749-1755. 
[29] Weinstein, M. C., and R. J. Zeckhauser. 1974. Use Patterns for Depletable and Recycleable Resources, Symposium on the Economics of Exhaustible Resources, Review of Economic Studies, 41, 67-88.

[30] Wan, R., and J. R. Boyce. 2014. Non-Renewable Resource Stackelberg Games, Resource and Energy Economics, 37, 102-121.

[31] Wirl, F., and E. Dockner. 1995. Leviathan Governments and Carbon Taxes: Costs and Potential Benefits, European Economic Review, 39, 1215-1236. 